\title{
Modeling of random geometric errors in superconducting magnets with applications to the CERN Large Hadron Collider
}

\author{
P. Ferracin, W. Scandale, E. Todesco, and R. Wolf \\ CERN, LHC Division, CH-1211 Geneva, Switzerland \\ (Received 25 October 2000; published 22 December 2000)
}

\begin{abstract}
Estimates of random field-shape errors induced by cable mispositioning in superconducting magnets are presented and specific applications to the Large Hadron Collider (LHC) main dipoles and quadrupoles are extensively discussed. Numerical simulations obtained with Monte Carlo methods are compared to analytic estimates and are used to interpret the experimental data for the LHC dipole and quadrupole prototypes. The proposed approach can predict the effect of magnet tolerances on geometric components of random field-shape errors, and it is a useful tool to monitor the obtained tolerances during magnet production.
\end{abstract}

PACS numbers: 85.25.Ly, 85.70.Ay, 41.85.Lc, 29.27.Bd

\section{INTRODUCTION}

The magnetic field generated by superconducting magnets used in particle accelerators [1] is affected by deviations from the ideal shape, which must be kept within a few $10^{-4}$ times the main field to avoid accelerator performance limitations. Field quality is determined mainly by three contributions: namely, the geometric part given by the positioning of the conductors inside the ferromagnetic yoke [1], a persistent current effect due to cable magnetization [1-3], and the yoke saturation at high field [1]. In order to obtain an acceptable field quality, conductor positions must agree with the nominal design within less than $0.1 \mathrm{~mm}$ [4]. Indeed, a strong feedback between magnet designers and magnet producers is needed, both in the design phase [5] and during the production [6].

Field shape is expanded in a power series in the coordinates of the magnet cross section plane. The coefficients of this expansion, called normal and skew multipoles, feature random variations along the longitudinal axis of the magnet. These variations are usually neglected in beam dynamics analysis, where each magnet is characterized by the average of its multipoles along the axis. A set of magnets features a systematic component (average of the magnet averages) and a random part (rms of the magnet averages).

A correct estimate of the random errors due to the geometric part has been considered a challenging and relevant issue for both beam dynamics and quality control during the magnet production [7-9]. In this paper we disregard multipole variations due to the random fluctuations of the cable or yoke magnetization, and we focus on the spread in the multipoles induced by the geometric part. In previous works $[7,8]$, random geometric errors have been modeled through random variation of coil positions and dimensions: the amplitude of the random variations was fixed by $a$ priori considerations, and experimental data were used to work out fitting parameters that describe the degree and the type of asymmetries found in built magnets. In this paper we develop a complementary approach, where all the asymmetries are considered equally probable, and the amplitude of the random displacement becomes the main fitting parameter. This leads to the same estimate for the normal and skew components of the same order.

The proposed approach allows us to analytically derive very simple scaling laws for geometric random errors, based on simplifying assumptions on the coil geometry. This provides a useful explicit dependence of the scaling law parameters on the coil characteristics, such as the magnet type, the cable thickness, and the magnet aperture. The analytical method is cross-checked with numerical simulations based on a Monte Carlo, using a detailed model of the coil cross section. We then compare our estimates with the experimental data at room temperature, where the cable magnetization and yoke saturation are not present. These data agree with the multipole decay foreseen by our scaling law, thus validating the way of modeling random errors.

The main fitting parameter of the scaling law is the amplitude of the random displacement. The analysis of experimental data in this framework provides an estimate of the actual tolerances reached during magnet manufacturing. We also investigate the relationship between random variations along the axis of one magnet and random variations among the averages of a set of magnets.

The obtained scaling laws for the random geometric multipoles have two main applications. On the one hand, they provide a solid estimate that can be input in tracking codes to evaluate beam stability. Moreover, they can be used during magnet production to monitor the actual tolerances and to trace back specific features of a particular magnet. Here we give estimates and applications to three magnet designs (two dipoles and one quadrupole), but this approach can be used for any type of coil layout.

The structure of the paper is the following. The main Large Hadron Collider (LHC) dipoles are analyzed in Sec. II. Numerical simulations to work out the scaling law are given in Sec. II A. Experimental data are analyzed within this framework in Sec. II B, where we distinguish 
random variations along the axis of a single dipole (Sec. II B 1), and random variations from magnet to magnet (Sec. II B 2). The main LHC quadrupole is analyzed in Sec. III, which is structured in the same way as Sec. II. Analytical estimates and parametric dependence of random multipoles due to random block displacements for a generic magnet are given in Sec. IV. Conclusions are drawn in Sec. V. The analysis of the previous five-block design of the LHC dipole coil is given in Appendix A. Detailed analytical computations of random errors are given in Appendix B.

\section{ANALYSIS OF THE LHC MAIN DIPOLE}

\section{A. Scaling law for random block displacements}

Random fluctuations of cable positioning can be analyzed through Monte Carlo methods. A very simple approach [10] is to assume that the blocks of conductors are rigid, and that they can freely move in the magnet cross section plane. A block of conductors is characterized by 3 degrees of freedom: for instance, one can choose two coordinates for the baricenter and an angle to fix the inclination of the block with respect to the horizontal axis. A cross section of the six-block design of the LHC dipole [11] is shown in Fig. 1.

Each block baricenter is shifted in the cross-section plane by two orthogonal vectors whose amplitudes belong to a Gaussian distribution with $\mathrm{rms} d / \sqrt{3}$. Moreover, the block is rotated around its baricenter by an angle such that the block corners move according to the same Gaussian distribution with $d / \sqrt{3} \mathrm{rms}$ amplitude. With respect to Refs. $[7,8]$, we neglect block deformations, but we also include the possibility of block rotations around the baricenter. The multipolar expansion coefficients $b_{n}$ and $a_{n}$,

$$
B_{y}(x, y)+i B_{x}(x, y)=B_{0} \sum_{n}\left(b_{n}+i a_{n}\right)\left(\frac{x+i y}{R_{\mathrm{ref}}}\right)^{n-1},
$$

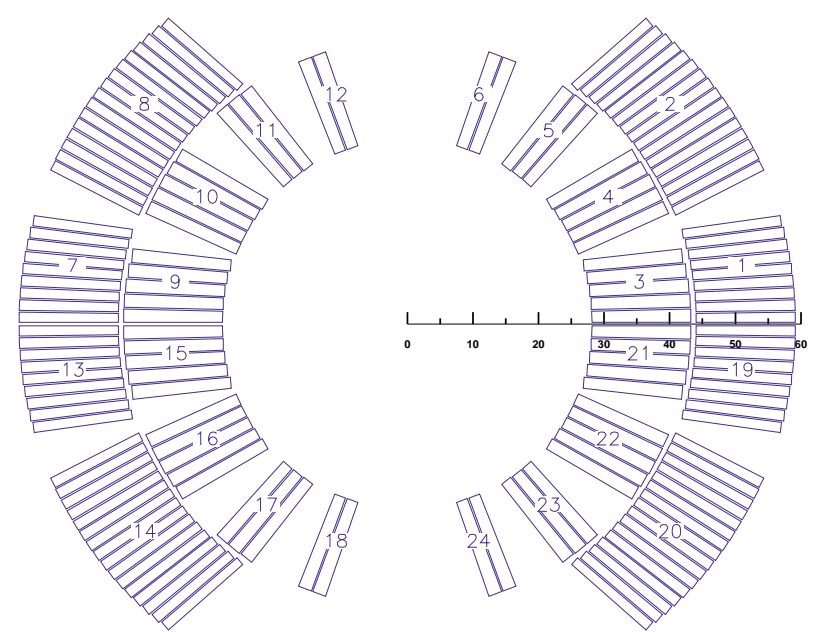

FIG. 1. (Color) Cross section of the six-block LHC dipole coil. of the obtained coil cross section are then evaluated. Here $B_{0}$ is the main field of the magnet. In this way, random displacements with rms amplitude $d$, equally shared by the block degrees of freedom, generate a distribution $b_{n}$ and $a_{n}$; the obtained multipole distributions are close to Gaussian, and the sigmas of normal and skew components $b_{n}$ and $a_{n}$ are rather similar. Numerical results are given in Fig. 2 (markers) for three different values of the rms amplitude $d$ ( 0.1 to $0.006 \mathrm{~mm})$. Throughout this paper, multipoles $b_{n}$ and $a_{n}$ are given in $10^{-4}$ units at the reference radius $R_{\text {ref }}=17 \mathrm{~mm}$. One can see that very regular patterns emerge from the numerical simulations; indeed, in a semilogarithmic scale numerical data are well fitted by a one-parameter family of second-order polynomials in the multipole order $n$; i.e.,

$$
\log \left[\sigma_{n}(d)\right]=a(d)+b n+c n^{2},
$$

where the parameter $a$ contains all the dependence on the rms displacement $d$. Equation (2) can be rewritten to the explicit dependence on $d$,

$$
\sigma_{n}(d)=d \alpha \beta^{n} \gamma^{n^{2}}
$$

i.e., the sigma of the random multipoles is proportional to the rms amplitude of the block displacement $d$, to a simple power law $\beta^{n}$ and a quadratic power law $\gamma^{n^{2}}$. The fitting constants for the five analyzed rms amplitudes, and their averages, are given in Table I.

The power law $\beta^{n}$ is due to the Biot-Savart law: a current line at a distance $r$ generates a magnetic field of order $1 / r$. This means that the magnetic field $B$ diverges on the position of the first conductor of the coil, whose distance from the magnet center is in the LHC dipole

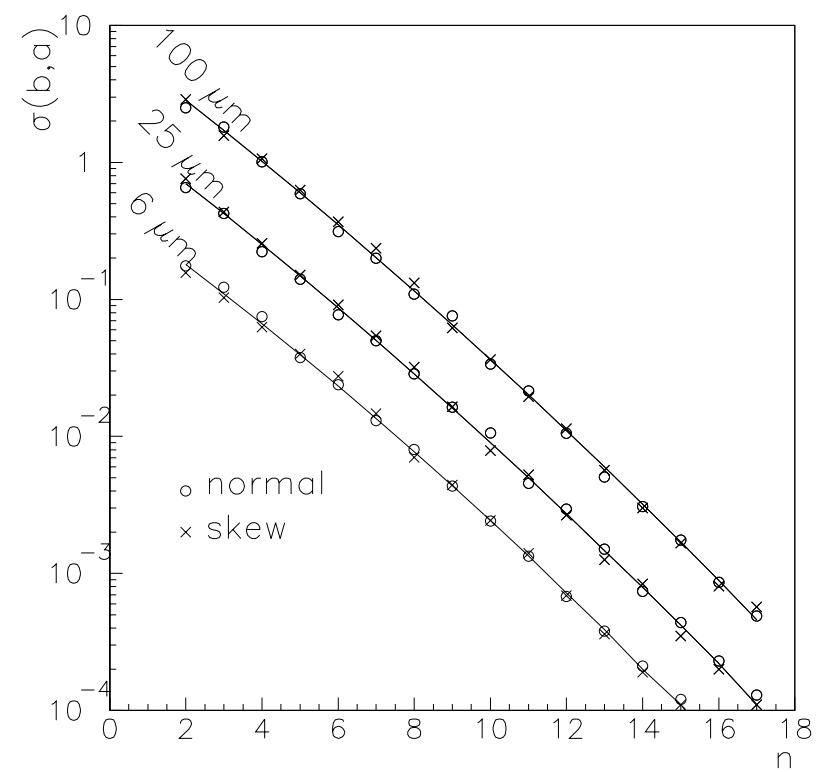

FIG. 2. Numerical estimate (markers) and parabolic fit (solid line) of the geometric random multipoles due to random block displacements of different amplitudes $d$ for the six-block coil of the main LHC dipole. 
TABLE I. Fitting constants $\alpha, \beta$, and $\gamma$ [see Eq. (3)] for random multipoles generated by random block displacements for the six-block coil of the main LHC dipole

\begin{tabular}{lccc}
\hline \hline$d(\mathrm{~mm})$ & $\alpha\left(\mathrm{mm}^{-1}\right)$ & $\beta$ & $\gamma$ \\
\hline 0.1 & 75.9 & 0.6180 & 0.9947 \\
0.05 & 73.6 & 0.6324 & 0.9933 \\
0.025 & 74.5 & 0.6209 & 0.9945 \\
0.0125 & 72.1 & 0.6281 & 0.9939 \\
0.00625 & 73.5 & 0.6339 & 0.9932 \\
Average & 73.9 & 0.6266 & 0.9939 \\
\hline \hline
\end{tabular}

$r_{0}=28 \mathrm{~mm}$. For this reason, the multipolar expansion of the field is convergent for $|r|=|x+i y|<r_{0}$ and the field intensity has the following upper bound:

$$
\left|B_{y}+i B_{x}\right|<\kappa \sum_{n} \frac{|x+i y|^{n}}{r_{0}^{n}} .
$$

Substituting it in Eq. (1) we obtain the upper bound for the harmonic $n$

$$
\left|b_{n}+i a_{n}\right|<\kappa^{\prime}\left(\frac{R_{\mathrm{ref}}}{r_{0}}\right)^{n} .
$$

Assuming that this convergence condition on the multipolar coefficients holds also for their $\operatorname{rms} \sigma\left(b_{n}\right)$ and $\sigma\left(a_{n}\right)$, one obtains a rough estimate of the parameter $\beta=R_{\text {ref }} / r_{0}=17 / 28=0.607$ that is indeed rather close to the numerical value 0.626 (see Table I).

The nonlinear term $\gamma^{n^{2}}$ provides a correction to the power law that gives a faster decrease of the higher order terms $(\gamma<1)$. In general, the larger the blocks, the faster the reduction of the high order multipoles with respect to the power law (this can also be seen by a direct comparison of the five-block design with the six-block design of the LHC dipole; see Appendix A).

In Sec. IV we develop an analytical model based on a simplified geometry of the coil; in this case one can derive analytical estimates for the random errors that agree well with the numerical results and that give some analytical insight on the dependence of random errors on magnet parameters.

\section{B. Analysis of experimental data}

Several prototypes of the LHC dipoles have been constructed and tested in the past 10 years [12]. Here we analyze the experimental data relative to four $15 \mathrm{~m}$ long final prototypes [13]. Field quality is measured at room temperature in the collared coils and in the assembled cold mass, with a low current (10 A). The sensitivity of these measurements is of the order of $10^{-7}$ with respect to the main field (i.e., 0.001 units in $10^{-4}$ ). Measurements are taken along 20 consecutive positions with a $750 \mathrm{~mm}$ rotating coil. The first and last are discarded in our analysis since they are affected by end effects. Therefore, one has
18 data along the magnet axis: we compute the best estimates of the average and of the rms. With 18 data, the rms estimate has a relative error of $30 \%$ (with a $95 \%$ confidence limit).

One has two sets of data for each aperture, which are analyzed independently. Sigmas of corresponding normal and skew multipoles are then averaged, thus reducing the error estimate to $17 \%$. One can check that the variation of the multipoles along the axis is not affected by the yoking procedure, since the main contribution comes from the collared coil. Data relative to four collared coils of the LHC dipole prototypes are analyzed.

\section{Multipole variations along the axis}

We first analyze the variation of the multipoles along the axis. The sigmas of these variations for the first final prototype MBP2N2 are shown in Fig. 3. We consider the average between the two apertures, normal and skew components, separately. One can see that experimental data agree extremely well with the decay worked out through the Monte Carlo method, up to multipoles of order 10 and higher. This means that the hypothesis of the random displacements of the blocks is a very good approximation to modelize random geometric errors. On the other hand, experimental data prove to be extremely sensitive. In our case, the feed down of $b_{11}$ on $b_{10}$ and $a_{10}$ is used to work out the magnetic center, and therefore the sigmas of the multipole of order 10 are zero (see Fig. 3). Experimental data are consistent with rigid random displacements of blocks with rms of the order of $0.025 \mathrm{~mm}$ [see Eq. (3)]. This agrees with the specified tolerances on mechanical components.

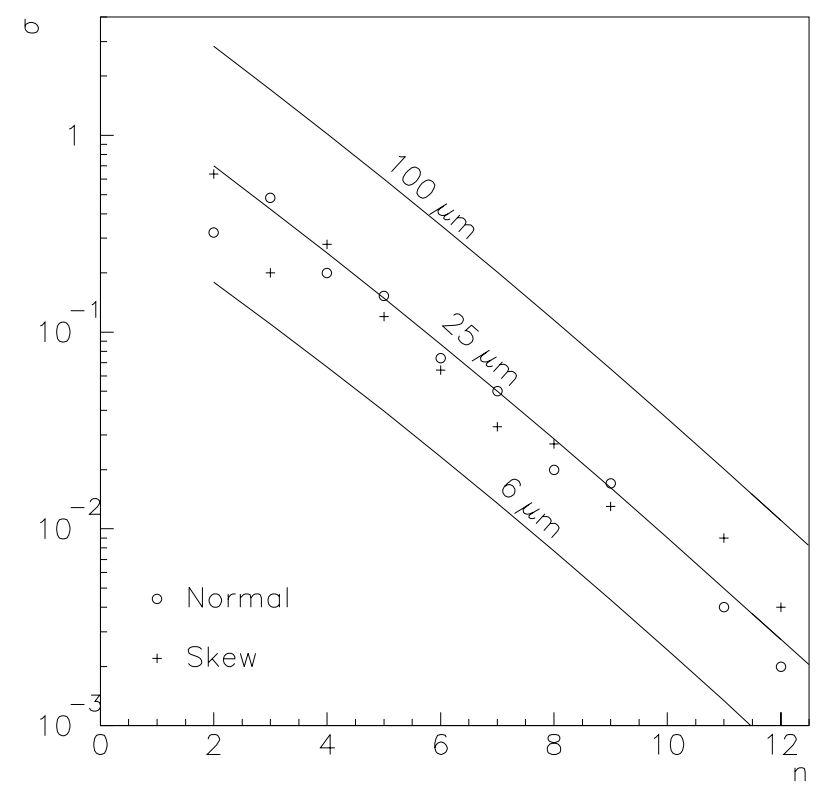

FIG. 3. rms of the multipoles along the axis for the LHC dipole prototype MBP2N2 (markers) and scaling law for different amplitudes of random displacements $d$ (solid line). 


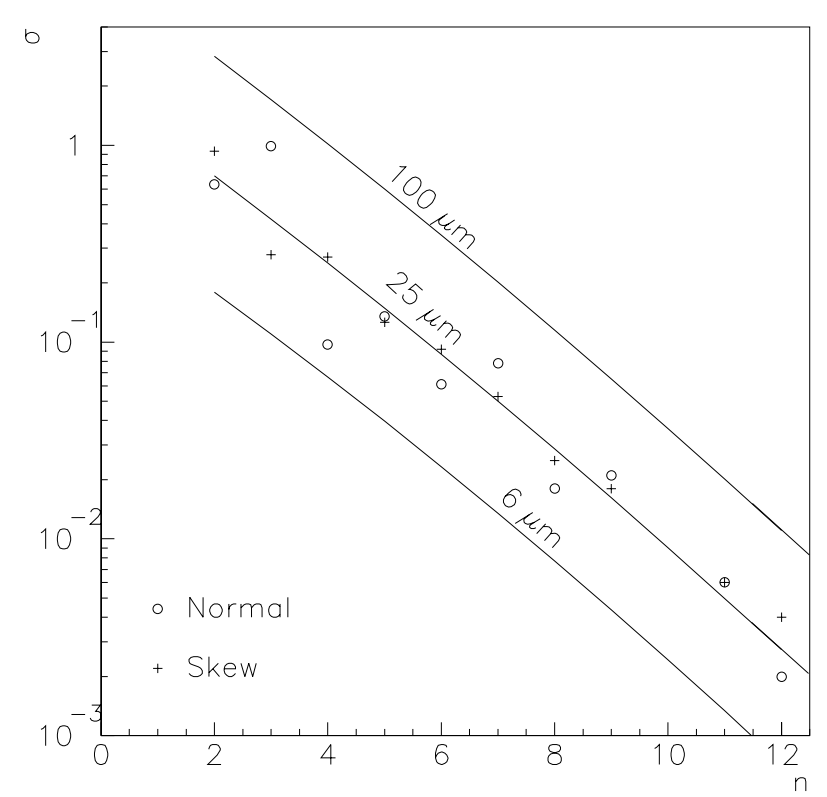

FIG. 4. rms of the multipoles along the axis for the LHC dipole prototype MBP2O1 (markers) and scaling law for different amplitudes of random displacements $d$ (solid line).

Our approach also allows detection of specific features of the assembled magnet: for example, in Fig. 4 we show the data of the prototype MBP2O1 that was assembled [14] with shims between collars and coils whose thickness varies along the axis up to $\pm 0.06 \mathrm{~mm}$. This specific shimming was used to follow the shape of the coil, to keep the azimuthal prestress as homogeneous as possible along the axis. Shimming usually affects the allowed multipoles if the dipole symmetry is preserved. In this case, shimming mainly affects the sextupole component $b_{3}$, as can be seen in a graph where experimental data are compared to the scaling law (see Fig. 4).

\section{Multipole variations between different magnets}

If each magnet were composed by cross sections that belong to a same distribution, with each multipole characterized by an average and a sigma, the variation of the multipoles along the axis of a single magnet would be larger than the variation of the averages of each magnet. Indeed, assuming a Gaussian distribution and a magnet composed by $N$ uncorrelated sections, the sigma of the averages would be $1 / \sqrt{N}$ times the sigma along the axis. Therefore, the sigma along the axis would overestimate the random component relevant for beam dynamics.

On the other hand, magnets produced with different tooling, components, and assembling procedures would be composed by cross sections belonging to different distributions, characterized by different averages and sigmas. In this case the sigma of the averages of all magnets can be higher than the sigma along the axis. This could happen also to magnets belonging to the same production line, due to drifts in components induced by the wear of the tooling.

Here we analyze the sigma of the averages of four collared coils (eight apertures) of the first LHC dipole final prototypes. The collared coils have been produced by three factories (two by Alstom-Jeumont and one by Noell and Ansaldo), using different tooling and components. The sigma of these averages is plotted together with our scaling law in Fig. 5. One observes much higher values, especially for the allowed multipoles $b_{3}, b_{5}, b_{7}$, and $b_{9}$, that are on the curve corresponding to a random displacement of $0.1 \mathrm{~mm}$. This is due mainly to relevant differences in the thickness of the coil shims used to impose the azimuthal prestress. Indeed, such shims determine the coil azimuthal length, which is strongly correlated to allowed multipoles. The other multipoles, with the exception of $a_{2}$ and $a_{4}$, have values that agree with a random displacement of approximately $0.025 \mathrm{~mm}$ rms amplitude. More dispersion is observed since a small number of apertures is considered.

One should conclude that, at this early stage of the dipole production, the random component is larger than the variation of the multipoles along the axis of a single magnet. In particular, our graphs indicate that an improved control of the coil azimuthal length should be employed to reduce the allowed multipoles.

The experimental data of the rms of the multipole averages show relevant differences between normal and skew components of the same order. This means that random movements are not equally shared by all the symmetries. One could include this effect, introducing additional fitting parameters as proposed in Refs. [7,8], but statistics on a wider set of magnets should be available.

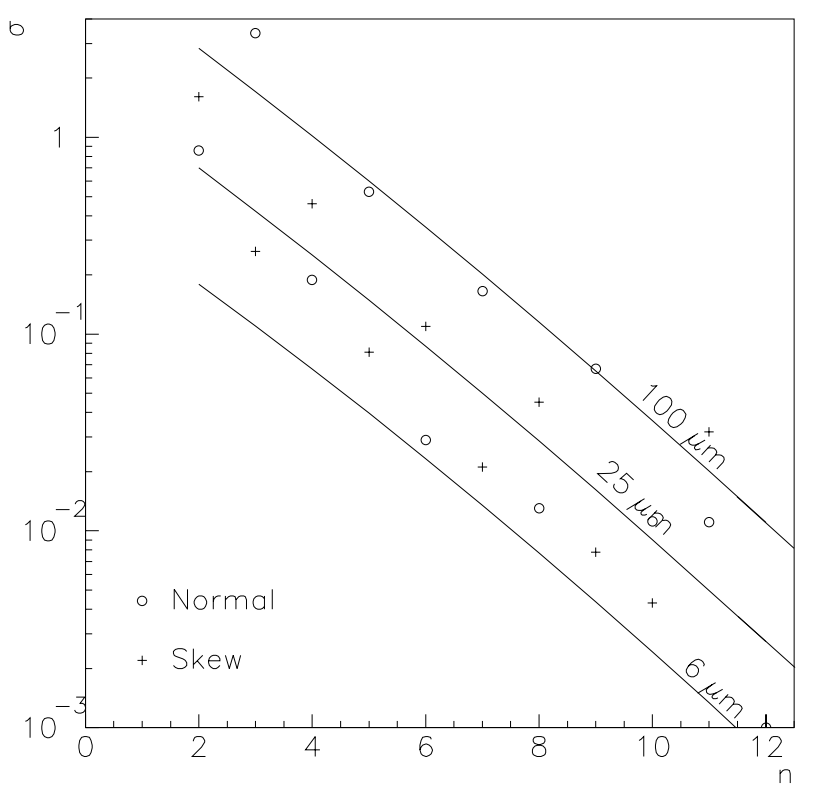

FIG. 5. rms of the multipole averages for the first four LHC dipole prototypes (markers) and scaling law for different amplitudes of random displacements $d$ (solid line). 


\section{ANALYSIS OF THE LHC MAIN QUADRUPOLE}

\section{A. Scaling law for random block displacements}

The main LHC quadrupole is characterized by a fourblock coil, disposed on two layers (see Fig. 6). Also in this case, we carried out a Monte Carlo analysis of the effect of small rigid random displacements of the block positions, assuming a Gaussian distribution of rms amplitude $d$. The sigmas of the obtained multipole distributions are shown in Fig. 7 (markers). Also in this case, one observes a very

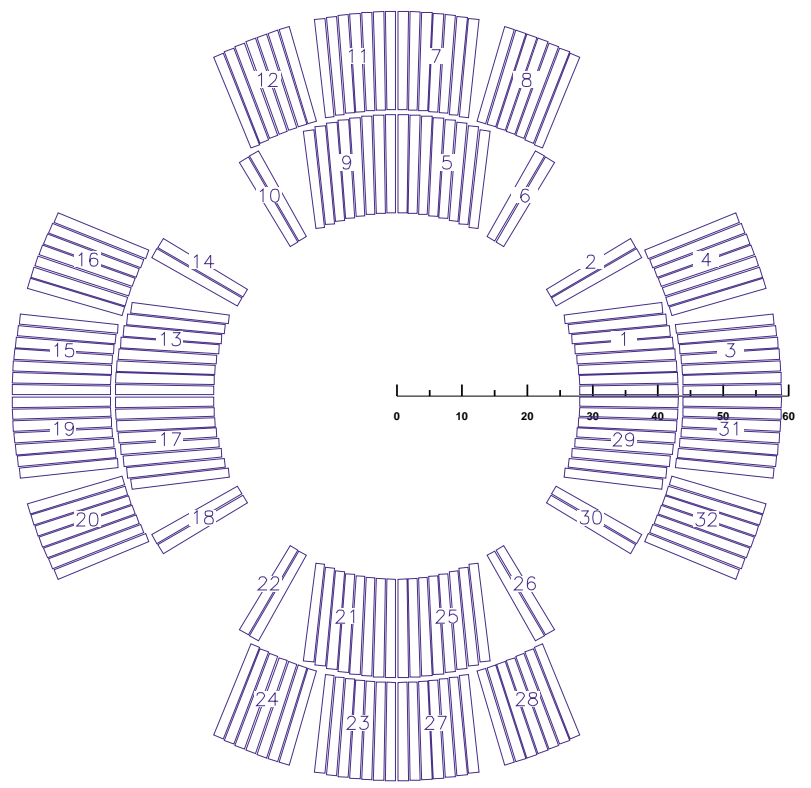

FIG. 6. (Color) Cross section of the four-block LHC quadrupole coil.

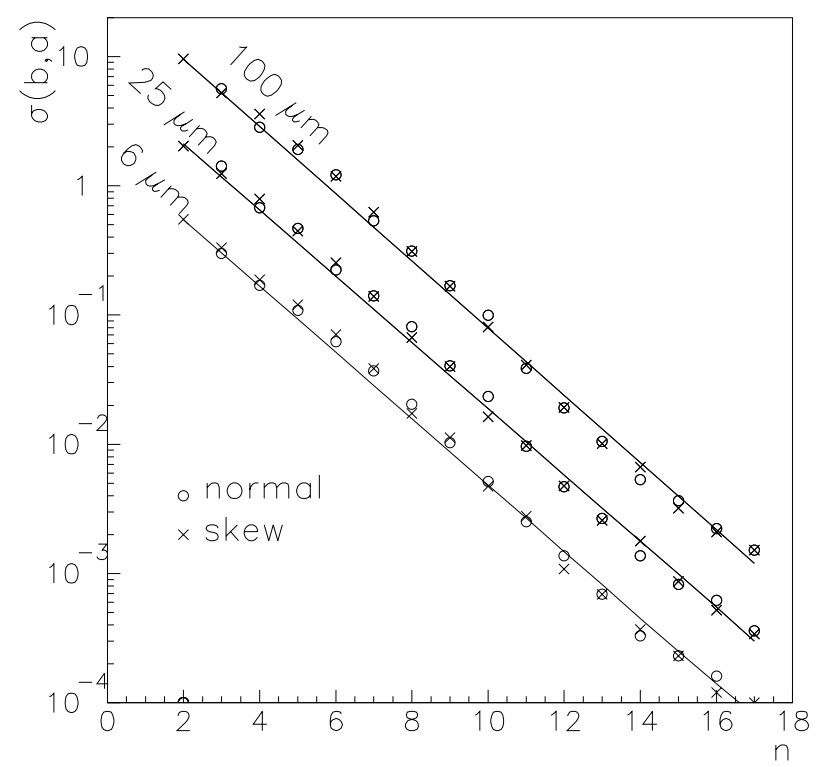

FIG. 7. Numerical estimate (markers) and parabolic fit (solid line) of the geometric random multipoles due to random block displacements of different amplitudes $d$ for the four-block coil of the main LHC quadrupole.
TABLE II. Fitting constants $\alpha$ and $\beta$ [see Eq. (6)] for random multipoles generated by random block displacements for the four-block coil of the main LHC quadrupole.

\begin{tabular}{lcc}
\hline \hline$d(\mathrm{~mm})$ & $\alpha\left(\mathrm{mm}^{-1}\right)$ & $\beta$ \\
\hline 0.1 & 316 & 0.5495 \\
0.05 & 269 & 0.5559 \\
0.025 & 274 & 0.5546 \\
0.0125 & 283 & 0.5546 \\
0.00625 & 285 & 0.5534 \\
Average & 285 & 0.5536 \\
\hline \hline
\end{tabular}

regular pattern, and a linear interpolation in the semilogarithmic scale takes into account numerical data. The best fit shown in Fig. 7 (solid lines) corresponds to a simple power law

$$
\sigma_{n}(d)=d \alpha \beta^{n}
$$

fitting constants worked out for different values of the amplitude rms $d$ are given in Table II. In this case the power law provides a good fit of experimental data. Moreover, one has a factor of 4 higher value of the constant $\alpha$ with respect to the dipole case: this means that the same displacements produce 4 times higher variations of the rms multipoles. This feature can be explained by our analytical model given in Sec. IV. This is not a concern for beam dynamics since multipolar errors have to be integrated over the quadrupole length $(3.2 \mathrm{~m})$ that is nearly 5 times smaller than the dipole length.

\section{B. Analysis of experimental data}

Three quadrupole prototypes have been built at Saclay [15]. Magnetic measurements at room temperature and low current $(12.5 \mathrm{~A})$ have been carried out using a rotating coil of $750 \mathrm{~mm}$ length. Multipole coefficients are measured in five consecutive positions along the magnet axis [16]. The first and last are discarded since they are affected by end effects. The obtained rms are affected by a large error (70\% with $2 \sigma$ confidence level, $40 \%$ when the average rms between two apertures is considered), but can still be used in our analysis.

\section{Multipole variations along the axis}

The rms of the multipoles along the axis of the first quadrupole prototype SSS3 versus the scaling law [Eq. (6)] is shown in Fig. 8. One observes that the measured sigmas are consistent with rigid random movements of the blocks of $0.025 \mathrm{~mm}$, such as in the case of the LHC dipoles. The observed difference between experimental data and scaling laws in the slope of the multipole decay could be statistically nonsignificant, and should be verified by a larger sample of data. 


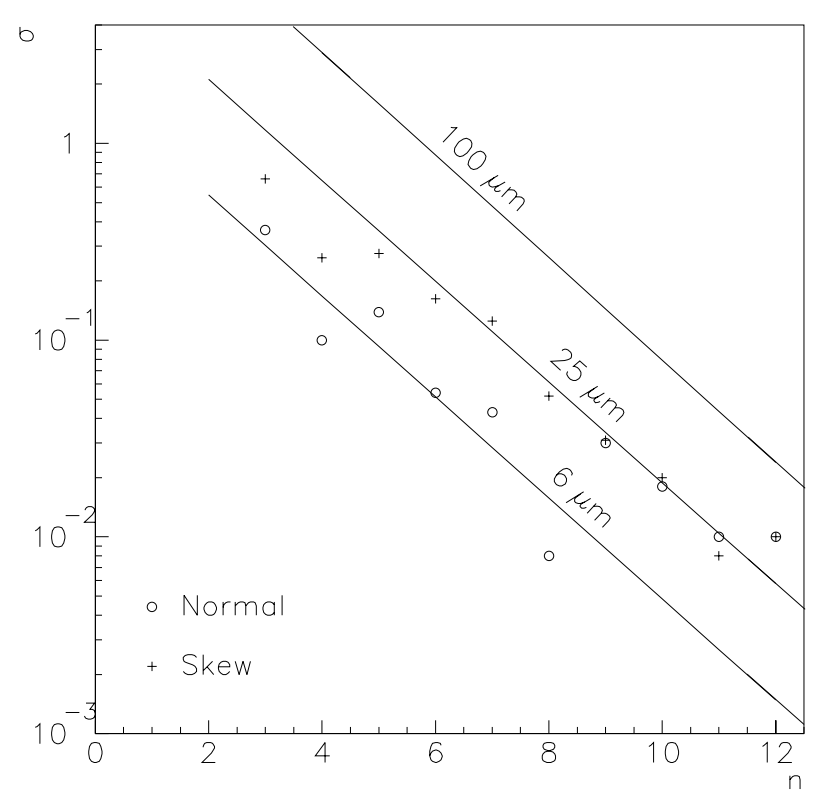

FIG. 8. rms of the multipoles along the axis for the LHC quadrupole prototype SSS3 (markers) and scaling law for different amplitudes of random displacements $d$ (solid line).

\section{Multipole variations between different magnets}

Contrary to the dipole case, all the quadrupole prototypes were produced in the same laboratory. The analysis of the rms of the averages of the six apertures (see Fig. 9) shows that variations from magnet to magnet are somewhat larger than the variations along the axis. This effect is particularly relevant in $b_{6}$ and $a_{4}$. Indeed, the values are much closer to the sigma along the axis than in the case of the

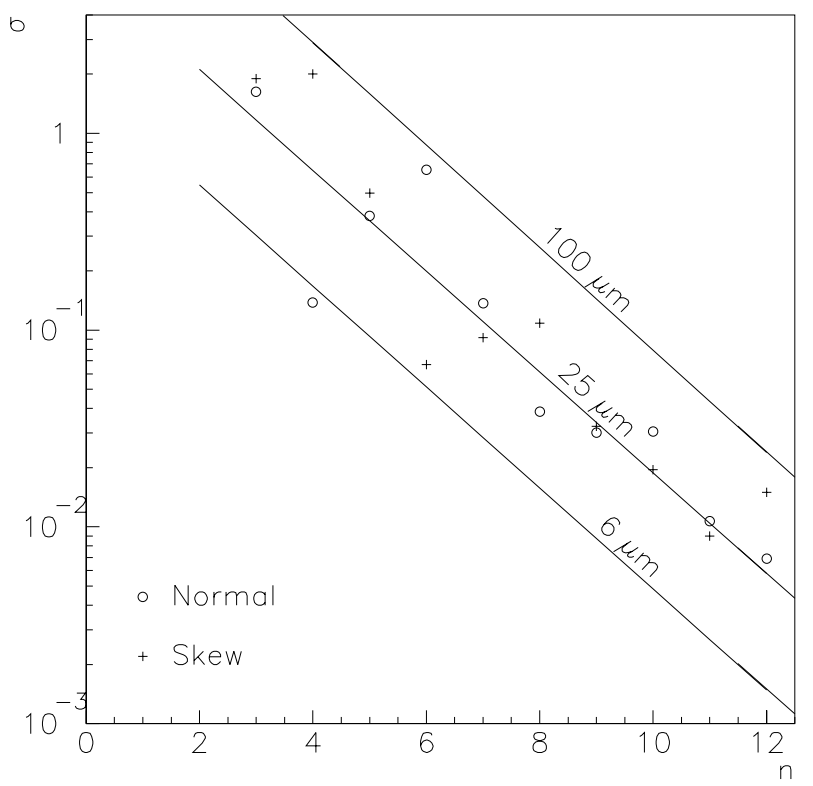

FIG. 9. rms of the multipole averages for the first three LHC quadrupole prototypes (markers) and scaling law for different amplitudes of random displacements $d$ (solid line). dipoles, where different factories were producing different prototypes. With the exception of the aforementioned multipoles, the sigmas of the averages correspond to random displacements of around $0.035 \mathrm{~mm}$. The multipole decay agrees well with our scaling law. The unexpectedly large values of $a_{4}$ and $b_{6}$ correspond to small values of $b_{4}$ and $a_{6}$, respectively. This means that a particular symmetry / antisymmetry of the magnet is excited, and the corresponding antisymmetry/symmetry for the same multipolar order is not. Therefore, our hypothesis of a complete randomization of the coil displacements is not verified in this case. Indeed, one has to point out the rather high error associated with the rms estimate due to poor statistics.

\section{ANALYTICAL ESTIMATE OF MULTIPOLES DUE TO RANDOM DISPLACEMENTS}

Here we give analytical formulas for multipoles generated by random displacements, assuming simple geometries for the magnet coil. We analyze a generic $2 N$ pole magnet $(N=1$ for a dipole, $N=2$ for a quadrupole, and so on), consisting of $4 N$ poles. We make the following assumptions: (i) Each pole is a sector of radii $r_{1}$ and $r_{2}$, and whose aperture is $\pi /(3 N)$ : in this way the first allowed multipole $b_{3 N}$ is zero (see, for instance, Ref. [1]); (ii) the current density in each pole is constant; (ii) the contribution of transport currents only is considered (no iron yoke); (iv) the sector can undergo only rigid translations.

The first assumption neglects the coil geometry that is usually made of more than one layer, and the presence of copper wedges that separate conductor blocks. The second one neglects the small radial gradient in the current density that is due to the cable keystoning. The contribution of iron can be considered in the usual mirror approximation: explicit formulas can be found in Refs. [10,17], where we also prove that rotations of the sectors can be neglected in a first approximation.

Under these simplifying hypotheses (see Appendix B for detailed computations), one obtains the following estimate of the rms $\sigma_{n}$ of the multipole of order $n$ due to a random displacement of rms $d$ :

$$
\sigma_{n} \propto d \frac{r_{1}}{\sqrt{N m}} \frac{N-2}{R_{\mathrm{ref}}^{N}\left(r_{1}^{2-N}-r_{2}^{2-N}\right)}\left(\frac{R_{\mathrm{ref}}}{r_{1}}\right)^{n} F\left(\frac{n+1}{6 m N}\right),
$$

where $N$ denotes the magnet type $(N=1$ for a dipole, $N=2$ for a quadrupole, and so on), $R_{\text {ref }}$ is the reference radius where the multipoles are rescaled, $r_{1}$ and $r_{2}$ are the inner and the outer radii of the coil, and $m$ is the number of subsectors that can move independently (i.e., the number of blocks). The function $F$ is defined as $F(x) \equiv$ $\sin (\pi x) /(\pi x)$.

The main dependence of $\sigma$ versus $n$ is a power law $\beta^{n}$ where $\beta=R_{\text {ref }} / r_{1}$, which corresponds to the power 
law that can be naively derived from upper bounds on the Taylor series of the multipoles (see Sec. II A). The constant in front of the power law

$$
\alpha(N) \propto \frac{r_{1}}{\sqrt{N m}} \frac{N-2}{R_{\text {ref }}^{N}\left(r_{1}^{2-N}-r_{2}^{2-N}\right)}
$$

features an interesting explicit dependence on the magnet type: for instance, for a dipole, $N=1$, one has

$$
\alpha(1) \propto \frac{r_{1}}{\sqrt{m_{1}}} \frac{1}{R_{\text {ref }}\left(r_{1}-r_{2}\right)},
$$

while, for a quadrupole, performing the limit $N \rightarrow 2$, one has

$$
\alpha(2) \propto \frac{r_{1}}{\sqrt{2 m_{2}}} \frac{1}{R_{\mathrm{ref}}^{2}\left(\log r_{1}-\log r_{2}\right)} .
$$

The ratio between the dipole and the quadrupole term is

$$
\frac{\alpha(2)}{\alpha(1)}=\sqrt{\frac{2 m_{2}}{m_{1}}} \frac{r_{1}-r_{2}}{R_{\text {ref }}\left(\log r_{1}-\log r_{2}\right)} ;
$$

for the LHC case one finds a ratio of around 3, in agreement with the numerical estimate $\alpha(2) / \alpha(1)=3.9$ (see the previous section).

The last term, $F[(n+1) /(6 N m)]$, in Eq. (7) is an oscillating term whose absolute value is bounded by 1 , and that decreases for high multipoles with $1 / n$ : therefore, it becomes negligible for high $n$ with respect to the main term $\beta^{n}$. Indeed, for low $n$ one can make a Taylor expansion for $x \ll 1$,

$$
\log F(x)=\log \frac{\sin \pi x}{\pi x} \approx \log \left(1-\frac{\pi^{2} x^{2}}{6}\right) \approx-\frac{\pi^{2} x^{2}}{6},
$$

and, therefore,

$$
F\left(\frac{n+1}{6 N m}\right)=\exp \left(-\frac{\pi^{2}}{6} \frac{(n+1)^{2}}{36 N^{2} m^{2}}\right)=\gamma^{(n+1)^{2}} .
$$

For the LHC dipole one can take $\mathrm{Nm}=6$ and, therefore, $\gamma=0.997$ in agreement with the numerical value $\gamma=$ 0.994 given in Sec. II A. For the quadrupole one can use $N m=8$, obtaining $\gamma=0.9994$ to be compared to $\gamma=$ 1.0000 found with numerical simulations.

\section{CONCLUSIONS}

In this paper we have modeled random errors in superconducting magnets due to the geometric component, i.e., to small discrepancies in the coil position with respect to the nominal design. Under the hypothesis that blocks undergo random movements that preserve their shape, we use a Monte Carlo method to work out a numerical estimate of the rms values of the multipoles. The obtained numerical values show very simple patterns that can be fitted by scaling laws. We propose a power law plus a nonlinear correction for the main LHC dipole coil, and a simple scaling law for the main LHC quadrupole. An analytical estimate based on simplifying assumptions supports these scaling laws, also providing a useful analytical insight on the parametric dependence.

The outlined methods can be used to provide a realistic estimate of the geometric part of the random errors. Moreover, it gives a link between random errors and the corresponding rms amplitudes of the random block displacements. Therefore, it can be used as a tool to monitor the actual tolerances in the coil cross section during magnet production.

Comparison with experimental data for the first prototypes confirm the estimated decay of the random multipoles. The rms of the multipoles along the axis of a same magnet agree with random block displacements of $0.025 \mathrm{~mm} \mathrm{rms}$, for both the dipole and the quadrupole. The rms of the averages of the analyzed magnets are higher, especially in the case of the dipoles produced by three different manufacturers at the very early stage of the production. The relationship between the variation of the multipoles along the axis and the variation of the multipoles over a set of magnet is also discussed. Our approach can be generalized to other coil cross sections.

\section{ACKNOWLEDGMENTS}

We wish to thank O. Pagano for providing the warm magnetic measurements of the main LHC dipoles used in this analysis. Simulations to evaluate the magnetic field and the multipolar errors have been carried out through ROXIE [18]: we wish to acknowledge S. Russenschuck for providing us the input files and for his kind help. Warm magnetic measurements of the main LHC quadrupoles have been carried out at the CEA in Saclay: we wish to thank J.-M. Rifflet, M. Peyrot, and F. Simon for providing the data and for helpful discussions.

\section{APPENDIX A: FIVE-BLOCK LHC DIPOLE}

The case of the five-block coil cross section previously used in the LHC dipole prototypes has also been analyzed, using the same techniques described in Sec. II A. The result of the Monte Carlo simulation is showed in Fig. 10, where data relative to three rms amplitudes $d$ are shown. Also in this case, numerical results (markers) are very well fitted by a second-order polynomial in a semilogarithmic scale. The same power law with a nonlinear correction [see Eq. (3)] holds, with rather similar values of the constants $\alpha, \beta$, and $\gamma$ (see Table III).

The main difference is in the constant $\gamma$ that in the five-block coil provides a more rapid decay of the high order multipoles; we already pointed out in Sec. II A that this is due to the larger size of the coil blocks. Indeed, this effect is totally irrelevant from the point of view of beam 


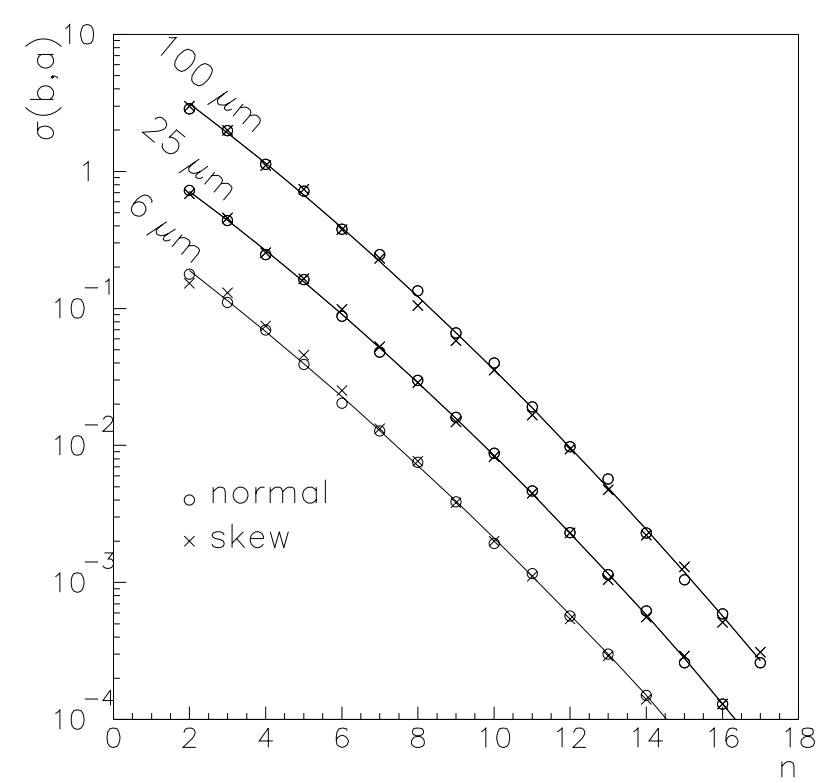

FIG. 10. Numerical estimate (markers) and parabolic fit (solid line) of the geometric random multipoles due to random block displacements of different amplitudes $d$ for the five-block coil of the main LHC dipole.

dynamics: the two designs can be considered equivalent as far as random multipoles are concerned.

The comparison of the scaling law for the five-block coil with experimental data relative to the $10 \mathrm{~m}$ long magnet prototype MBL1N2 is given in Fig. 11. Also in this case, the rms of the multipoles along the axis are consistent with random displacements of the block with an rms amplitude of around $0.025 \mathrm{~mm}$.

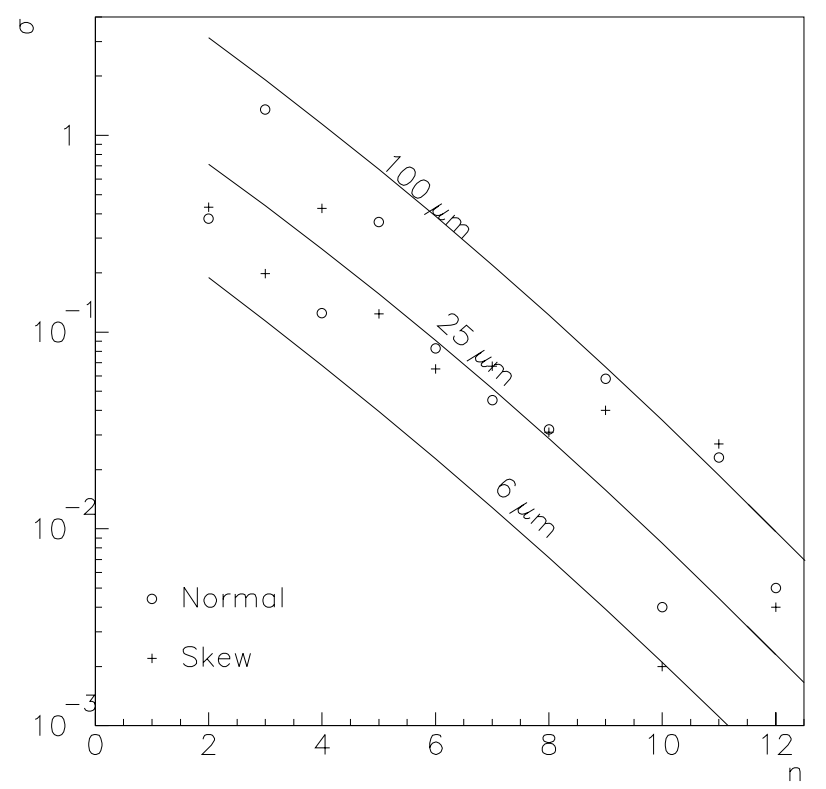

FIG. 11. rms of the multipoles along the axis for the LHC dipole prototype MBL1N2 (markers) and scaling law for different amplitudes of random displacements $d$ (solid line).
TABLE III. Fitting constants $\alpha, \beta$, and $\gamma$ [see Eq. (3)] for random multipoles generated by random block displacements for the five-block coil of the main LHC dipole

\begin{tabular}{lccc}
\hline \hline$d(\mathrm{~mm})$ & $\alpha\left(\mathrm{mm}^{-1}\right)$ & $\beta$ & $\gamma$ \\
\hline 0.1 & 79.6 & 0.6397 & 0.9907 \\
0.05 & 75.9 & 0.6353 & 0.9911 \\
0.025 & 71.3 & 0.6457 & 0.9903 \\
0.0125 & 70.5 & 0.6295 & 0.9917 \\
0.00625 & 79.1 & 0.6281 & 0.9919 \\
Average & 75.3 & 0.6353 & 0.9912 \\
\hline \hline
\end{tabular}

\section{APPENDIX B: ANALYTICAL ESTIMATE OF RANDOM ERRORS}

Let us first consider the case of a line current of intensity $I$, located at the point $z_{c}=x_{c}+i y_{c}$; this generates a field at the point $z=x+i y$ corresponding to the Biot-Savart law,

$$
B_{y}+i B_{x}=\frac{\mu_{0} I}{2 \pi} \frac{1}{z-z_{c}}=-\frac{\mu_{0} I}{2 \pi} \sum_{n=1}^{\infty} \frac{z^{n-1}}{z_{c}^{n}},
$$

where we expanded the fraction as a Taylor series. This has to be compared to the standard multipolar expansion

$$
B_{y}+i B_{x}=\sum_{n=1}^{\infty} C_{n} z^{n-1}=B_{N} R_{\mathrm{ref}}^{N-1} \sum_{n=1}^{\infty} c_{n} \frac{z^{n-1}}{R_{\mathrm{ref}}^{n-1}}
$$

where $B_{N}=C_{N}$ is the component of the main field and $R_{\text {ref }}$ is the reference radius that defines $c_{n}=b_{n}+i a_{n}$. One gets the expression for $c_{n}$ :

$$
c_{n}=-\frac{1}{B_{N} R_{\mathrm{ref}}^{N-n}} \frac{\mu_{0} I}{2 \pi} \frac{1}{z_{c}^{n}} .
$$

A small variation of the conductor position $\Delta z_{c}$ leads to a variation in $c_{n}$,

$$
\Delta c_{n}=\frac{n}{B_{N} R_{\mathrm{ref}}^{N-n}} \frac{\mu_{0} I}{2 \pi} \frac{\Delta z_{c}}{z_{c}^{n+1}} .
$$

We now assume that the magnet is composed by $4 N$ sectors of angular amplitude $\pi /(3 N)$, so that the first order allowed multipole is set to zero (see, for instance, [1]). We now integrate all the $\Delta c_{n}$ contributions over the sector that carries a constant current density $J$,

$$
\begin{aligned}
\int \Delta c_{n} d s= & \frac{n \Delta z_{c}}{B_{N} R_{\mathrm{ref}}^{N-n}} \frac{\mu_{0} J}{2 \pi} \\
& \times \int_{r_{1}}^{r_{2}} \int_{0}^{\pi / 3 N} \frac{e^{-i \phi(n+1)}}{\rho_{c}^{n+1}} \rho d \rho d \phi,
\end{aligned}
$$

where we assumed that the block undergoes a rigid translation and therefore $\Delta z_{c}$ is the same for all the sector points. We also assume that $\Delta z_{c}$ has zero average and standard deviation $d$, and performing the integral we obtain the rms of the multipoles 


$$
\sigma\left(c_{n}\right)=\frac{n}{B_{N} R_{\mathrm{ref}}^{N-n}} \frac{\mu_{0} J}{2 \pi} \frac{r_{1}^{1-n}-r_{2}^{1-n}}{n-1} F\left(\frac{n+1}{6 N}\right) \frac{\pi d}{6 N},
$$

$\sigma\left(c_{n}\right)=\frac{r_{1}}{3 R_{\mathrm{ref}}^{N}} \sqrt{\frac{4}{3 N m}} \frac{N-2}{r_{1}^{2-N}-r_{2}^{2-N}}\left(\frac{R}{r_{1}}\right)^{n} F\left(\frac{n+1}{6 N}\right)$

where we defined

$$
F(x) \equiv \frac{\sin \pi x}{\pi x}
$$

We now assume a constant current density $J$, replacing it by the total current $I$ flowing in the sector divided by the sector area $\pi\left(r_{2}^{2}-r_{1}^{2}\right) /(3 N)$,

$$
\begin{aligned}
\sigma\left(c_{n}\right)= & \frac{n}{n-1} \frac{1}{B_{N} R_{\mathrm{ref}}^{N-n}} \frac{\mu_{0} I}{2 \pi} \frac{r_{1}^{1-n}-r_{2}^{1-n}}{r_{2}^{2}-r_{1}^{2}} \\
& \times F\left(\frac{n+1}{6 N}\right) d .
\end{aligned}
$$

The main field $B_{N}$ can be evaluated in a similar way by performing the integral over the sectors of the expression given in Eq. (B2),

$$
B_{N}=3 \sqrt{3} N \frac{\mu_{0} I}{\pi^{2}\left(r_{2}^{2}-r_{1}^{2}\right)} \frac{r_{1}^{2-N}-r_{2}^{2-N}}{N-2} .
$$

Substituting in Eq. (B8) we finally obtain for a single sector

$$
\sigma\left(c_{n}\right)=\frac{r_{1}}{3 \sqrt{3}} \frac{N-2}{R^{N}\left(r_{1}^{2-N}-r_{2}^{2-N}\right)}\left(\frac{R}{r_{1}}\right)^{n} F\left(\frac{n+1}{6 N}\right) .
$$

We now assume that each sector is made up of $m$ blocks, each of them moving independently; the final sigma for the magnet is $\sqrt{4 N / m}$ the above sigma,
[1] K.-H. Mess, P. Schmüser, and S. Wolff, Superconducting Accelerator Magnets (World Scientific, Singapore, 1996).

[2] B. J. Holzer, Part. Accel. 55, 215-225 (1996).

[3] R. Wolf, IEEE Trans. Magn. 28, 374-377 (1992).

[4] W. Scandale, E. Todesco, and P. Tropea, IEEE Trans. Appl. Supercond. 10, 73-76 (2000).

[5] S. Peggs, Part. Accel. 54, 83-92 (1996).

[6] M. D. Anerella, D. H. Fisher, E. Sheedy, and T. McGuire, IEEE Trans. Magn 32, 2059-2064 (1996).

[7] J. Herrera et al., IEEE Trans. Nucl. Sci. 32, 3689-3691 (1985).

[8] J. Herrera et al., in Proceedings of the 1987 Particle Accelerator Conference, Washington (IOP, New York, 1987), pp. 1477-1479.

[9] R. Gupta, Part. Accel. 55, 129-139 (1996).

[10] W. Scandale, E. Todesco, and R. Wolf, IEEE Trans. Appl. Supercond. 10, 93-97 (2000).

[11] P. Fessia et al., IEEE Trans. Appl. Supercond. 10, 65-68 (2000).

[12] C. Wyss, in Proceedings of the VII European Particle Accelerator Conference, Vienna (Austrian Academy of Sciences Press, Vienna, 2000), pp. 149-153.

[13] M. Modena et al., in Proceedings of the VII European Particle Accelerator Conference (Ref. [12]), pp. 2148-2150.

[14] G. Spigo (private communication).

[15] T. Tortschanoff et al., in Proceedings of the VII European Particle Accelerator Conference (Ref. [12]), pp. 21722174.

[16] E. Todesco et al., in Proceedings of the VII European Particle Accelerator Conference (Ref. [12]), pp. 2166-2168.

[17] R. Wolf, CERN LHC-MMS Internal Note No. 99-03, 1999.

[18] S. Russenschuck, CERN Report No. 99-02, 1999. 